\title{
Converting scores between the PANSS and SAPS/SANS beyond the positive/negative dichotomy
}

Stéphanie Grot ${ }^{1,2}$, Charles-Édouard Giguère ${ }^{1}$, Salima Smine ${ }^{1}$, Violaine Mongeau-Pérusse $^{2}$, Dana Diem Nguyen ${ }^{3}$, Adrian Preda ${ }^{4}$, Stéphane Potvin ${ }^{1,2}$, Theo G.M. van Erp ${ }^{5,6}$, FBIRN ${ }^{7}$, Pierre Orban ${ }^{1,2, *}$

1. Research Center of the Montreal Mental Health University Institute, Montreal, Quebec, Canada

2. Department of Psychiatry and Addictology, University of Montreal, Montreal, Quebec, Canada

3. Department of Pediatrics, University of California Irvine, Irvine, California, USA

4. Department of Psychiatry and Human Behavior, University of California Irvine, Irvine, California, USA

5. Clinical Translational Neuroscience Laboratory, Department of Psychiatry and Human Behavior, University of California Irvine, Irvine, California, USA

6. Center for the Neurobiology of Learning and Memory, University of California Irvine, Irvine, California, USA

7. Function Biomedical Informatics Research Network, USA

* Correspondence: pierre.orban@umontreal.ca

1 Centre de recherche de l'Institut Universitaire en Santé Mentale de Montréal 7331, rue Hochelaga

Montréal, QC, H1N 3V2

Canada 


\begin{abstract}
Background: Previous work provided conversion equations for overall indices of positive and negative symptomatology between the two most widely used scales to assess symptom severity in schizophrenia, namely the Positive and Negative Syndrome Scale (PANSS) and the Scales for the Assessment of Positive/Negative Symptoms (SAPS/SANS). Our objective was to provide such conversion equations for subdomains of positive and negative symptomatology in order to better account for the diversity of symptom profiles in schizophrenia.
\end{abstract}

Method: Symptoms severity was assessed using both the PANSS and SAPS/SANS in 205 patients with schizophrenia. Two exploratory factor analyses combining items from both scales were first performed separately in the positive and negative symptom domains. For each identified factor, linear regression analyses were then conducted to obtain conversion equations from the PANSS to the SAPS/SANS and vice versa. Linear regression model estimation was performed on $80 \%$ of the data, and reliability was then evaluated on the $20 \%$ remaining data using intra-class correlation coefficient between the original and predicted scores. This procedure was repeated 100 times with random samplings for each factor cross-scale conversion.

Results: Three-factor solutions were favored both in the positive and negative symptom domains. Based on the nature of items that strongly loaded on the different factors, positive factors were termed 'Hallucinations', 'Delusions' and 'Disorganization', while negative factors were associated with 'Expressivity', 'Amotivation' and 'Cognition'. Intra-class correlation coefficients between the original and predicted scores were good to excellent $(0.68-0.87)$ for all regressions, but for the cognition factor which were deemed as low $(0.25$, $0.26)$.

Conclusion: The symptom subdomains identified by the decomposition of the positive and negative domains were consistent with current descriptions of symptom dimensions in schizophrenia. With the exception of the cognition subdomain, symptom severity scores can be converted with good accuracy between scales, beyond the positive/negative symptom dichotomy. Conversion equations are implemented in an R Shiny app to facilitate their use by the clinical research community.

Keywords: conversion equation, data pooling, psychosis dimensions, rating scale, schizophrenia, symptoms heterogeneity 


\section{Background}

Various scales and instruments are available to researchers and clinicians alike to objectively measure the severity of symptoms in schizophrenia and other psychotic disorders. The multiplicity of symptom rating scales not only hampers efficient communication between clinicians, but also undermines cross-study comparison, metaand mega-analyses, and retrospective data aggregation in research [1]. Such is for instance the case when pooling multi-site data through SchizConnect, a platform that provides access to disparate neuroimaging datasets of which related symptom data in schizophrenia were obtained using different scales across datasets [2]. In this age of open-access and big data, it is thus imperative to develop a bridge system across clinical scales that may facilitate the translation of symptom data in schizophrenia between studies and datasets.

Qualitative approaches, such as the comparison of scale scores with outcomes or creation of binary remission criteria, have been proposed [1]. However, these translation methods do not preserve the quantitative information of symptom ratings. Provided different scales correlate well and thereby have good concurrent validity, it is instead conceivable to derive formulae that estimate scores on one scale from scores on another. The Positive and Negative Syndrome Scale (PANSS, [3]) as well as the Scale for the Assessment of Positive Symptoms (SAPS, [4]) combined with the Scale for the Assessment of Negative Symptoms (SANS, [5]) are two of the most widely used rating symptom scales to assess schizophrenia. Research shows that they measure broadly similar constructs and their overall indices for positive and negative symptomatology to be well correlated $(r=0.70-0.91)$, though less so when using scores from different raters [1,6]. Using symptom ratings obtained on both the PANSS and SAPS/SANS scales in a relatively large sample of schizophrenia patients, van Erp and colleagues demonstrated that regression-based equations can satisfactorily convert ratings of overall indices of positive and negative symptomatology between scales [7]. Intraclass correlations between the original scores and those predicted by the conversion equations were good to excellent $(\mathrm{ICC}=0.65-0.91)$ [8], with slightly better performance for negative compared to positive symptoms.

The dichotomy between sets of positive and negative symptoms has largely guided the development of clinical scales such as the PANSS or SAPS/SANS, and consequently clinical practice and research, since Crow's groundbreaking model positing schizophrenia as a two-syndrome disease four decades ago [9]. The recognition of negative symptoms (e.g., alogia and apathy) beyond positive symptoms (e.g., hallucinations, delusions and thought disorders) that were the focus of interest at the time was an important step towards better accounting for the phenotypically heterogeneous nature of schizophrenia. Yet, this distinction still failed to capture the full diversity of symptom profiles observed in this psychiatric disorder. Accordingly, positive symptoms have since then been subdivided into florid psychotic and disorganized dimensions $[10,11]$ while negative symptoms have been further decomposed into dimensions related to diminished expression and avolition $[12,13]$.

Our objective was to provide conversion equations between the PANSS and SAPS/SANS beyond the previously reported conversion of overall indices of positive and negative 
symptomatology [7]. To this end, we leveraged the same dataset of symptom severity ratings obtained with both scales in a community-based sample of schizophrenia patients, as part of Phase 3 of the Function Bioinformatics Research Network (FBIRN) multi-center brain imaging project [14]. Factors that combined items from both scales were first identified, separately in the positive and negative domains. Regression-based equations were then computed between scales for each identified factor, and their reliability assessed. Findings reveal that subdomains of positive (hallucinations, delusions and disorganization) and negative (expressivity, amotivation and cognition) symptomatology common to both scales are broadly consistent with the literature, and that ratings conversion between scales can be performed accurately for all symptom subdomains but the so-called cognition one.

\section{Method}

\section{Participants}

A sample of 205 individuals suffering from schizophrenia (156/49 males/females, mean age $\pm \mathrm{SD}=39.5 \pm 11.6$ ) was recruited from 7 sites as part of the Phase 3 of the Function Biomedical Informatics Research Network (FBIRN) study (Table 1). Schizophrenia diagnosis was determined by experienced clinical raters using the Structured Clinical Interview for DSM-IV-TR Axis I Disorders (SCID-I/P) [15]. Diagnosis subtypes were distributed as follows: 146 paranoid, 7 disorganized, 36 undifferentiated, and 16 residual subtypes. Patients with schizoaffective disorder were excluded. Other exclusion criteria are described in length in previous studies [7,16]. Clinical assessments of symptoms included the Positive and Negative Syndrome Scale (PANSS, [3]) as well as the Scale for the Assessment of Positive Symptoms (SAPS, [4]) and the Scale for the Assessment of Negative Symptoms (SANS, [5]), rated based on the severity of symptoms over the last month. The same rater administered all scales for a given patient, and rating standardization was promoted across sites through cross-site training. All participants provided written informed consent, including permission to share de-identified data within the FBIRN consortium as approved by the Institutional Review Boards of the University of California Irvine, the University of California Los Angeles, The University of California San Francisco, Duke University, University of North Carolina, University of New Mexico, University of Iowa and University of Minnesota.

Table 1 here

Symptom rating scales

The PANSS [3] is composed of 30 items organized into three distinct subscales that respectively measure positive, negative and general psychopathology symptom domains. Each item is rated on a severity scale that ranges from 1 (absent) to 7 (extreme). Although this standard scoring procedure was used in the present work, it should be noted that items scores may be recoded from 0 to 6 ([17]). Seven items (1-7) define the positive scale, and another seven (8-15) the negative scale, with scores thus ranging from 7 to 49 for each 
scale. The remaining 16 items define the general psychopathology scale, which will be ignored in this study as it lacks proper correspondence in the SAPS/SANS [1].

The SAPS [4] includes 34 items that focus on the positive symptom domain and define 4 subscales measuring hallucinations (items 1-6), delusions (8-19), bizarre behavior (21-24) and positive formal thought disorder (26-33). Each item is rated on a severity scale that ranges from 0 (absent) to 5 (most severe). In addition, each subscale has its own global score that similarly ranges from 0 to 5 (from absent to most severe) and rates the overall severity of each symptom domain. The SAPS total (composite) score is the sum of all but the global items, while the SAPS summary (global) score is the sum of the 4 global items. The SANS [5] comprises 25 items that specifically assess the negative symptom domain and define 5 subscales measuring affective flattening or blunting (items 1-7), alogia (9-12), avolition-apathy (14-16), anhedonia-asociality (18-21) and attention (23-24). Rating is performed similarly to that of the SAPS, with the SANS summary score corresponding to the sum of 5 global items.

\section{Statistical analyses}

Analyses were performed using base R 3.5 [18] as well as the psych version 1.8.4 [19] and the ICC.Sample.Size version 1.0 [20] R packages.

\section{Factor analyses}

Two separate exploratory factor analyses were conducted on respectively the positive and negative items, the first one combining the 7 positive PANSS items with the 4 global SAPS items and the second one pooling together the 7 negative PANSS items with the 5 global SANS items. Both models were estimated with a principal component analysis using an oblimin rotation that accounts for possible intercorrelations between factors. The number of factors being retained was determined by visually inspecting the scree plot of eigenvalues on the number of components (factors) and selecting only those largest components with corresponding eigenvalues above 1 . Items within a factor were only included in further analyses if they had a positive loading above 0.4 on that factor.

\section{Conversion equations}

For each factor, we computed the average score for the items loading on that factor, separately for PANSS and SAPS/SANS items. Average item scores were also calculated for total (or summary) positive and negative scores of the PANSS and SAPS/SANS. Different conversion equations from the PANSS to SAPS/SANS, and vice versa, were then computed for every factor or total score. Simple linear regressions were adjusted in a training sample, then mean squared error (MSE) and the intraclass correlation (ICC) between original and converted scores were obtained in a test sample. A power analysis determined the minimum test sample size needed for a medium ICC to be significant with sufficient power (ICC $=0.5, \alpha=0.05, \beta=0.1$ ). An $80-20 \%$ balance between the training and test samples conservatively satisfied this condition, corresponding to 164 and 41 patients in the training and test samples, respectively. For each total or factor score, this 
sampling procedure was repeated 100 times with randomly selected training data sets in order to obtain estimates of the $2.5 \%, 50 \%$ and $97.5 \%$ quantiles of ICCs and MSEs. All conversion equations reported in this study correspond to models that produced the ICC closest to the median ICC across sampling schemes. ICCs were interpreted as poor $(<0.40)$, fair (0.40-0.59), good (0.60-0.74) or excellent (0.75-1) [8].

\section{Results}

\section{Factor analyses}

Positive correlations were observed between most individual items of the PANSS and global items of the SAPS/SANS, with higher correlations found within (positive or negative) domains (Fig. 1a). Inspection of the scree plots for the positive and negative factor analyses favored 3-factor solutions in both cases (Fig. 1b). At least one item from the PANSS and one from the SAPS/SANS satisfied the inclusion criterion (positive loading $>0.4$ ) in every factor. Positive correlations were present between factors, both in the positive (range: 0.07-0.30) and negative (0.20-0.43) symptom domains. Based on the nature of items that strongly loaded on the different factors, positive factors were termed 'Delusions' (Delusions, Grandiosity, Suspiciousness and Hostility items in PANSS; Delusions global item in SAPS), 'Disorganization' (Disorganization item in PANSS; Bizarre behavior and Thought disorder global items in SAPS) and 'Hallucinations' (Hallucinations item in PANSS; Hallucinations global item in SAPS), while negative factors best corresponded to 'Expressivity' (Blunted affect, Poor rapport and Lack of spontaneity items in PANSS; Affective flattening and Alogia global items in SANS), 'Amotivation' (Emotional withdrawal and Social withdrawal items in PANSS; Avolitionapathy and Anhedonia-asociality global items in SANS) and 'Cognition' (Abstract thinking item in PANSS; Attention global item in SANS) (Fig. 1c, Table 2).

Figure 1 here

Table 2 here

\section{Conversion equations}

Parameters of representative linear regression models used to convert total or factor scores from the PANSS to the SAPS/SANS, and vice versa, are given in Table 2. Median MSEs were fairly low (0.12-0.92) for all regressions but for the cognition factor $(1.38,2.15)$. MSE values corresponding to the $97.5 \%$ quantile were not much higher $(0.18-1.29)$, with large values for the regressions of the cognition factor $(2.02,2.72)$.

Median ICCs were good to excellent (0.68-0.87) for all regressions, at the exception of poor ICCs (0.25 and 0.26) in the case of the cognition factor (Fig. 2). ICC values corresponding to the $2.5 \%$ quantile remained good to excellent $(0.60-0.78)$, but for the 
regressions of disorganization (fair, 0.51) and cognition (poor, 0.10) factors. Over all regressions, there was an average ICC range of 0.22 between the $2.5 \%$ and $97.5 \%$ quantiles.

In order to facilitate the use of these regression-based conversion equations by the clinical research community, an interactive version is made available through an $\mathrm{R}$ Shiny app (https://github.com/pnplab/convert_app). It requires to install two $\mathrm{R}$ packages with the following commands in an $\mathrm{R}$ console:

install.packages("shiny")

install.packages("shinyjs")

Conversions are then performed with shiny::runGitHub("pnplab/convert_app").

Figure 2 here

\section{Discussion}

Concomitant factor analyses on PANSS and SAPS/SANS items revealed a series of factors with shared positively loaded items across scales, separately in the positive and negative domains. Three positive factors correspond to primary criteria for the diagnostic of schizophrenia in the DSM-5 [21], namely hallucinations, delusions and disorganization. Two negative factors relate to the description of diminished emotional expression and amotivation/avolition as the two most prominent negative symptoms in this prevailing diagnostic manual. A last so-called cognition factor was isolated in the cross-scale factor structure of negative symptoms. However, as discussed hereafter, the validity of this factor is however much questionable and the conversion between scales for this factor was inaccurate and unreliable. This is in sharp contrast with the five other positive or negative factors for which between-scale symptom rating conversion could be performed accurately and reliably.

\section{Subdomains of positive and negative symptomatology}

Our cross-scale decomposition of the PANSS and SAPS/SANS into distinct positive and negative symptom subdomains can be comprehended in light of qualitative comparison of the two scales [1] as well as numerous past factor analyses conducted on either scale $[22,23]$. In contrast to prior studies, it should be noted that our factor analyses investigated both scales concomitantly rather than separately, considered positive and negative symptoms separately, relied on global SAPS or SANS scores rather than on individual items, and excluded items from the PANSS general psychopathology subscale.

The distinction between reality distortion (combining hallucinations and delusions) and disorganization within positive symptoms has received much support from the triadic syndrome model $[10,11,24]$, for instance underlying the SAPS/SANS. This dissociation is also found in rating scales with a more comprehensive list of symptoms such as the PANSS, 
which is however often described through a 5 -factor model that includes excitement/mania and depression dimensions in addition to reality distortion, disorganization and negative dimensions when including items from the general psychopathology subscale [22]. Of note, the hostility item loads highly on the excitement/mania factor in PANSS 5-factor models, rather than being included in a delusion (reality distortion) factor as reported in the present findings. While factor analysis on either the SAPS/SANS or PANSS does not typically distinguish hallucinations and delusions within reality distortion as found in the present work, it is worth noting that such has been the case using the Schedule for Clinical Assessment in Neuropsychiatry (SCAN) $[25,26]$. This symptom dimension separation is also supported by the DSM-5 dimensional model, allowing better correspondence with categorical diagnoses and clinical treatment targets [27].

Consistent with our results, ample evidence supports the delineation of diminished expressivity (poverty of speech and affective flattening) and amotivation (avolition/apathy and asociality) as two key underlying subdomains of negative symptoms $[12,13,28]$. While the DSM-5 dimensional model only includes a single negative symptom item for pragmatic reasons, it does describe the negative symptom dimension as somewhat of a hybrid, referring to deficits in either of the two subdomains [27].

The third factor we identified based on negative symptoms alone included cognitionrelated items, namely attention in the SANS and abstract thinking in the PANSS. Cognitive impairment is now widely recognized as a core feature of schizophrenia [29,30], as acknowledged in the DSM-5 dimensional model [27]. Yet, past factor analysis on either the SAPS/SANS or PANSS did not uncover a purely cognitive dimension. Rather, poor attention and difficulty in abstract thinking are often part of a disorganization factor $[10,22]$. Accordingly, the inclusion of attentional impairment as a negative symptom in the SANS has been questioned [31]. Besides, it should be noted that the attention item in the PANSS is part of the general psychopathology subscale, and it was therefore not included in our factor analysis. More generally, cognitive assessment based on symptom rating scales such as the SAPS/SANS or PANSS is likely imperfect, and instead requires formal neuropsychological testing [29]. Moreover, attention and abstract thinking point to only two of the many nonsocial and social cognitive domains with compromised functioning in schizophrenia [29,30]. For all these reasons, we conclude that the so-called cognition factor evidenced here has poor face validity across scales and should therefore be considered with caution.

\section{Accuracy and reliability of conversion equations}

Regression-based equations to quantitatively convert symptom ratings between distinct clinical scales have been successfully derived not only in schizophrenia $[7,16]$ but also in other mental illnesses such as major depression [32,33]. Simple linear regression models adequately described the relationship between scales, with only trivial gains induced by more complex functions [32]. In keeping with these findings, we assessed conversion reliability by contrasting original and predicted scores for a subset of data independent from data used to derive regression equations. We further evaluated how variable reliability measures were by conducting similar regression analyses multiple times using different 
permutations of training and test data, thereby allowing to report representative conversion equations.

Using a slightly modified approach, we first replicated previous results showing that overall indices of positive and negative symptomatology can be accurately and reliably converted between the PANSS and SAPS/SANS using the same dataset [7]. Our main contribution was to show that the severity of various subdomains of positive and negative symptoms can be converted between those rating scales as accurately and reliably as overall indices. One notable exception relates to the inaccurate and unreliable conversion of the so-called cognition factor, the validity of which we have questioned. We thus strongly advice against using conversion equations for this specific factor. In order to facilitate the use of all other regression-based conversion equations by the clinical research community, an interactive version is made available through an R Shiny app. A first note of caution is however warranted: the conversion equation we provide are based on classical scoring of PANSS items from 1 to 7 . In case PANSS items are originally scored from 0 to 6 [17], individual item scores should be rescaled prior to using our conversion equations. Moreover, we underscore that the good accuracy and reliability of conversion equations applied to averages does not imply good translation at the idiographic level [33]. Hence, care should be exercised when using our conversion equations in a clinical context or when predicting individual cases using machine learning approaches in research.

\section{Limitations}

Inherent to the dataset are a number of limitations which we only briefly address here, as they have been previously discussed [7,16]. First, assessment of symptom severity by a unique rater in a single session likely leads to overestimate the accuracy of score conversion one would observe if ratings on the two scales were obtained by different raters [6]. Second, while the dataset rests on a community-based sample, all schizophrenia patients were psychiatrically stable with only mild to moderate symptom severity and no substance abuse. Hence, it is unknown how well the proposed conversion equations would perform for more severely ill or acute schizophrenia patients, given that our regression approach depends highly on the dispersion of symptom ratings. In the same line, positive and negative symptoms are present in other non-affective and affective psychotic disorders. Similar to schizophrenia, symptom ratings on the PANSS and SAPS/SANS appear highly correlated in bipolar disorder [34], however, it is unknown to what extent our specific conversion equations for subdomains of positive and negative symptomatology would generalize well to psychotic disorders other than prototypical stable schizophrenia. Finally, future work should investigate conversion equations with the Brief Psychiatric Rating Scale (BPRS, [35]), as this other symptom rating scale widely used to assess symptom severity in schizophrenia shows good correspondence with both the PANSS and SAPS/SANS [1].

\section{Conclusions}

In an era of open and big data, efficient pooling of quantitative symptom data from distinct rating scales would greatly facilitate retrospective aggregation of multi-site data as well as 
meta- and mega-analyses through collaborative efforts. Our work complements conversion equations for overall positive and negative symptom ratings between the PANSS and SAPS/SANS in schizophrenia [7]. Consistent with the idea that schizophrenia is phenotypically heterogeneous beyond the positive/negative dichotomy, we developed similarly accurate and reliable between-scale conversion equations for a number of relevant positive and negative symptom subdomains. This endeavour aligns well with the need for a more dimensional approach in the characterization of schizophrenia and other psychotic disorders, beyond categorical diagnosis [36]. Consequently, it will be essential to assess the usefulness of our conversion equations in various non-affective and affective psychotic disorders, in a transdiagnostic perspective.

\title{
Declarations
}

\author{
Abbreviations \\ BPRS, Brief Psychiatric Rating Scale; FBIRN, Function Biomedical Informatics Research \\ Network; ICC, intra-class correlation coefficient; MSE, mean squared error; PANSS, \\ Positive and Negative Syndrome Scale; SANS, Scale for the Assessment of Negative \\ Symptoms; SAPS, Scale for the Assessment of Positive Symptoms.
}

\section{Acknowledgments}

The authors are grateful to research subjects for their participation.

\section{Funding}

SG is supported by a doctoral fellowship of the Fonds de Recherche du Québec - Santé (FRQS). SP is holder of the Eli Lilly Canada Chair on Schizophrenia research. PO is supported by a salary award Junior 1 of the Fonds de Recherche du Québec - Santé (FRQS) and a Canadian Institutes of Health Research (CIHR) project grant (\#PJT-165910). This work was also supported by the National Institutes of Health grant numbers: NIH 1 U24 RR021992 (Function Biomedical Informatics Research Network) and NIH 1U24 RR025736-01 (Biomedical Informatics Research Network Coordinating Center).

\section{Ethics approval and consent to participate}

All participants provided written informed consent, including permission to share deidentified data as approved by the Institutional Review Boards of the University of California Irvine, the University of California Los Angeles, The University of California San Francisco, Duke University, University of North Carolina, University of New Mexico, University of Iowa and University of Minnesota. 


\section{Consent for publication}

Not applicable.

Availability of data and materials

Data are not publicly available because permission was not obtained from participants. Any question regarding the dataset may be addressed to TVE (tvanerp@uci.edu). Simulated data, code used to generate the reported results and figures, as well as interactive conversion equations through an $\mathrm{R}$ Shiny app are accessible on Github at https://github.com/pnplab/convert.

\section{Authors' contributions}

Conception and design: PO. Data acquisition: DDN, AP, TVE. Data analysis: CEG, SS, VMP, TVE, PO. Writing: SG, SP, PO. All authors read, commented and approved the final manuscript.

\section{Competing interests}

AP has served as a consultant for Boehringer-Ingelheim, GLG, and Guidepoint. Other others declare that they have no competing interests. 


\begin{tabular}{|l|l|}
\hline Demographics & Mean (SD) \\
\hline Age (years) & $39.5(11.6)$ \\
\hline Age at onset (years) & $21.8(7.5)$ \\
\hline Duration of illness (years) & $17.7(11.5)$ \\
\hline & \\
\hline Symptoms & \\
\hline & \\
\hline PANSS & $15.7(5.3)$ \\
\hline Positive & $14.9(5.9)$ \\
\hline Negative & $28.6(7.5)$ \\
\hline General & $59.1(15.4)$ \\
\hline Total & \\
\hline & \\
\hline SAPS & $6.0(6.1)$ \\
\hline Hallucinations & $8.2(7.8)$ \\
\hline Delusions & $1.3(1.7)$ \\
\hline Bizarre behavior & $1.3(1.7)$ \\
\hline Thought disorder & $16.8(14.2)$ \\
\hline Total (composite) & $5.7(3.7)$ \\
\hline Global (summary) & \\
\hline SANS & $6.2(6.8)$ \\
\hline Flat affect & $2.4(3.2)$ \\
\hline Alogia & $4.6(3.3)$ \\
\hline Apathy & $7.0(5.1)$ \\
\hline Anhedonia & $2.7(2.4)$ \\
\hline Attention & $23.0(14.6)$ \\
\hline Total (composite) & $7.8(4.7)$ \\
\hline Global (summary) & \\
\hline & \\
\hline & \\
\hline & \\
\hline & \\
\hline & \\
\hline & \\
\hline & \\
\hline
\end{tabular}

Table 1. Sample characteristics. More details can be found in previous studies $[7,16]$. 


\begin{tabular}{|c|c|}
\hline \multicolumn{2}{|c|}{ Scores computation } \\
\hline PANSS & SAPS/SANS \\
\hline \multirow[t]{2}{*}{ PANSS_positive $=$ mean $($ PANSS01-07) } & $\begin{array}{l}\text { SAPS_total = mean(SAPS01-06,08-19,21- } \\
24,26-33)\end{array}$ \\
\hline & SAPS_summary $=$ mean(SAPS07,20,25,34) \\
\hline \multirow[t]{2}{*}{ PANSS_negative $=$ mean $($ PANSS08-14) } & $\begin{array}{l}\text { SANS total = mean(SANS01-07,09-12,14- } \\
16,18-21,23-24)\end{array}$ \\
\hline & $\begin{array}{l}\text { SANS_summary }=\text { mean }(\text { SANS01,13,17,22, } \\
25)\end{array}$ \\
\hline PANSS_delusions $=$ mean $($ PANSS01,05-07) & SAPS_delusions $=$ SAPS20 \\
\hline PANSS disorganization $=$ PANSS02 & SAPS disorganization $=$ mean $($ SAPS 25,34$)$ \\
\hline PANSS hallucinations $=$ PANSS03 & SAPS hallucinations $=$ SAPS07 \\
\hline $\begin{array}{l}\text { PANSS_expressivity }= \\
\text { mean(PANSS08,10,13) }\end{array}$ & SANS_expressivity $=$ mean $($ SANS08,13) \\
\hline PANSS amotivation $=$ mean $($ PANSS09,11) & SANS amotivation $=$ mean $($ SANS17,22) \\
\hline PANSS_cognition $=$ mean $($ PANSS12 $)$ & SANS_cognition $=$ SANS25 \\
\hline \multicolumn{2}{|c|}{ Conversion equations } \\
\hline SAPS/SANS to PANSS & PANSS to SAPS/SANS \\
\hline $\begin{array}{l}\text { PANSS_positive }=1.499+1.079 * \\
\text { SAPS_total }\end{array}$ & $\begin{array}{l}\text { SAPS_total }=-0.543+0.528 * \\
\text { PANSS_positive }\end{array}$ \\
\hline $\begin{array}{l}\text { PANSS_positive }=1.325+0.631 * \\
\text { SAPS_summary }\end{array}$ & $\begin{array}{l}\text { SAPS_summary }=-0.637+0.918 * \\
\text { PANSS_positive }\end{array}$ \\
\hline $\begin{array}{l}\text { PANSS_negative }=1.016+0.962 * \\
\text { SANS_total }\end{array}$ & $\begin{array}{l}\text { SANS_total }=-0.370+0.721 * \\
\text { PANSS_negative }\end{array}$ \\
\hline $\begin{array}{l}\text { PANSS_negative }=0.953+0.753 * \\
\text { SANS_summary }\end{array}$ & $\begin{array}{l}\text { SANS_summary }=-0.363+0.883 * \\
\text { PANSS_negative }\end{array}$ \\
\hline $\begin{array}{l}\text { PANSS_delusions }=1.265+0.494 * \\
\text { SAPS_delusions }\end{array}$ & $\begin{array}{l}\text { SAPS_delusions }=-0.849+1.293 * \\
\text { PANSS delusions }\end{array}$ \\
\hline $\begin{array}{l}\text { PANSS_disorganization }=1.234+0.979 * \\
\text { SAPS_disorganization }\end{array}$ & $\begin{array}{l}\text { SAPS_disorganization }=-0.245+0.481 * \\
\text { PANSS_disorganization }\end{array}$ \\
\hline $\begin{array}{l}\text { PANSS_hallucinations }=1.235+0.859 * \\
\text { SAPS_hallucinations }\end{array}$ & $\begin{array}{l}\text { SAPS_hallucinations }=-0.773+0.941 * \\
\text { PANSS hallucinations }\end{array}$ \\
\hline $\begin{array}{l}\text { PANSS_expressivity }=0.977+0.752 * \\
\text { SANS expressivity }\end{array}$ & $\begin{array}{l}\text { SANS_expressivity }=-0.541+0.907 * \\
\text { PANSS_expressivity }\end{array}$ \\
\hline $\begin{array}{l}\text { PANSS_amotivation }=0.698+0.843 * \\
\text { SANS_amotivation }\end{array}$ & $\begin{array}{l}\text { SANS_amotivation }=0.430+0.677 * \\
\text { PANSS amotivation }\end{array}$ \\
\hline $\begin{array}{l}\text { PANSS_cognition }=2.455+0.436 * \\
\text { SANS_cognition }\end{array}$ & $\begin{array}{l}\text { SANS_cognition }=0.492+0.329 * \\
\text { PANSS_cognition }\end{array}$ \\
\hline
\end{tabular}

Table 2. Scores computation and conversion equations. Formulas are given to calculate total, summary and factor scores for positive and negative symptoms. Parameters of the most representative linear regression models to convert scores between the PANSS and SAPS/SANS are given, as is implemented in a Shiny $\mathrm{R}$ app (https://github.com/pnplab/convert_app). 
a

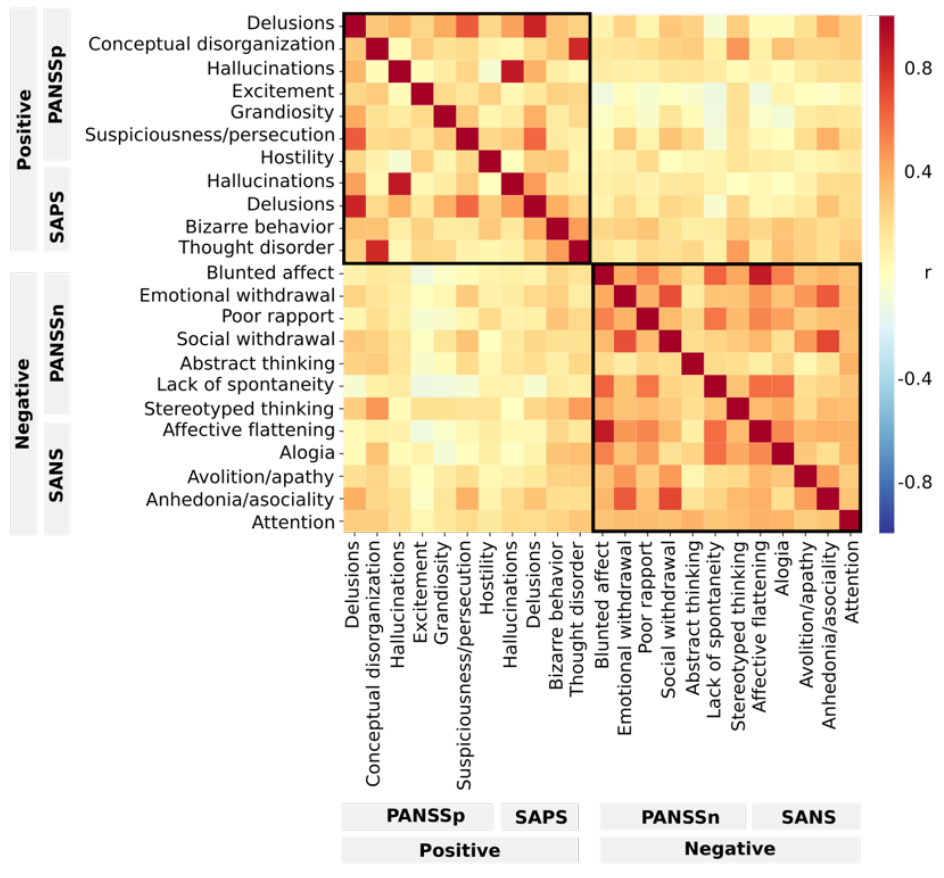

b
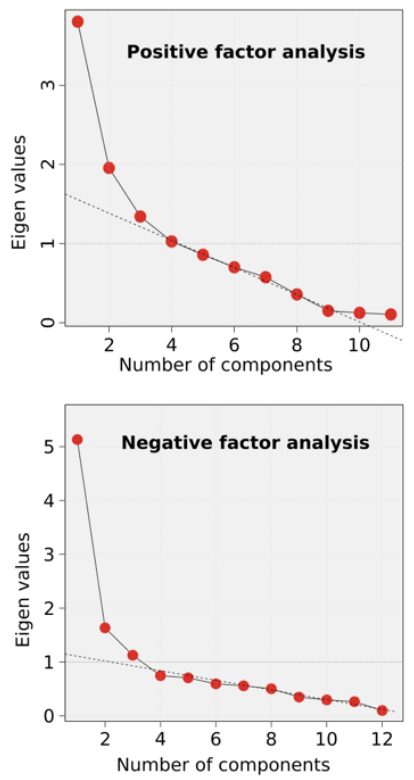

C
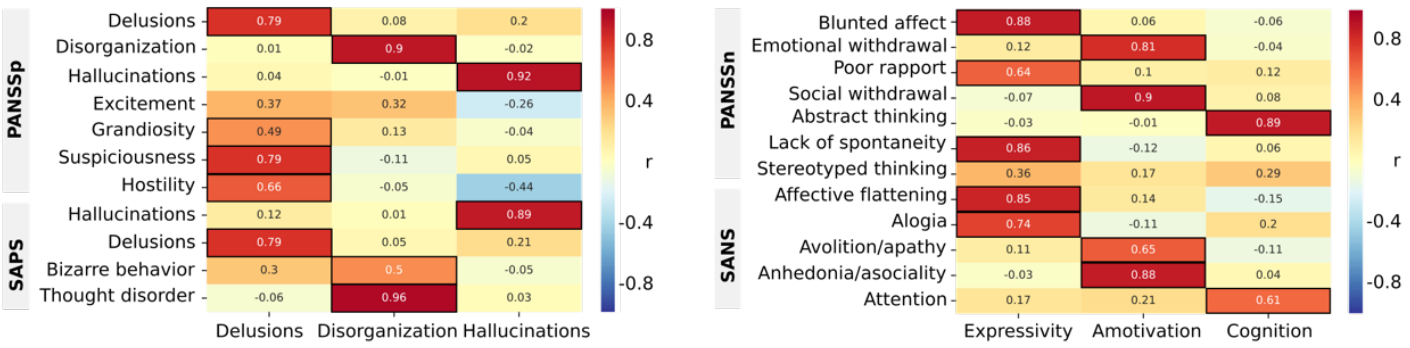

Fig. 1. Factor analysis. (a) Correlation matrix between all positive and negative PANSS items, and global positive and negative SAPS/SANS items. (b) Scree plots of the exploratory factor analyses on positive and negative symptoms. (c) Loadings of PANSS and SAPS/SANS items on the selected positive (delusions, disorganization, hallucinations) and negative (expressivity, amotivation, cognition) factors. 


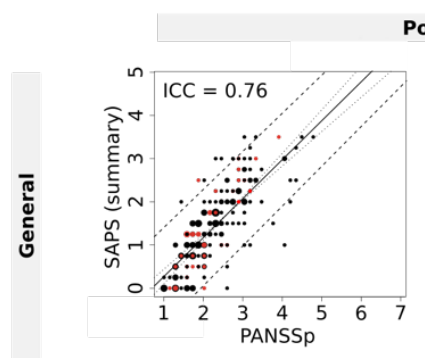

Positive

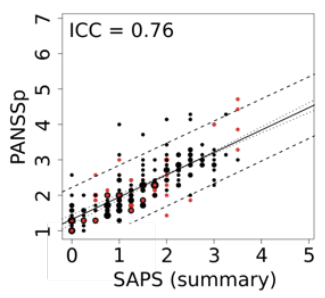

Positive
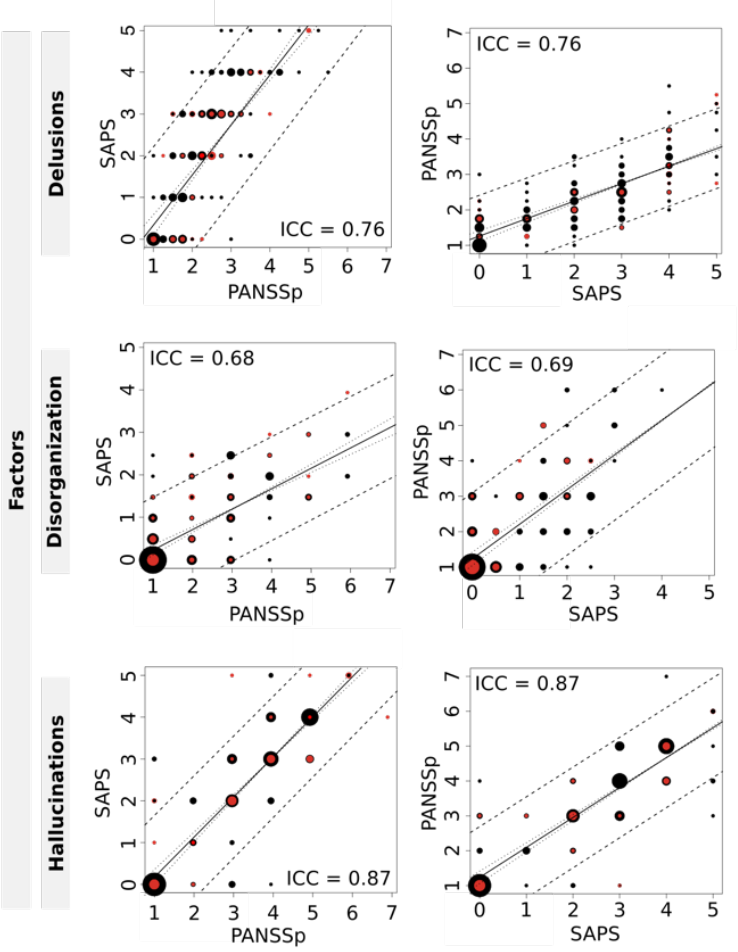
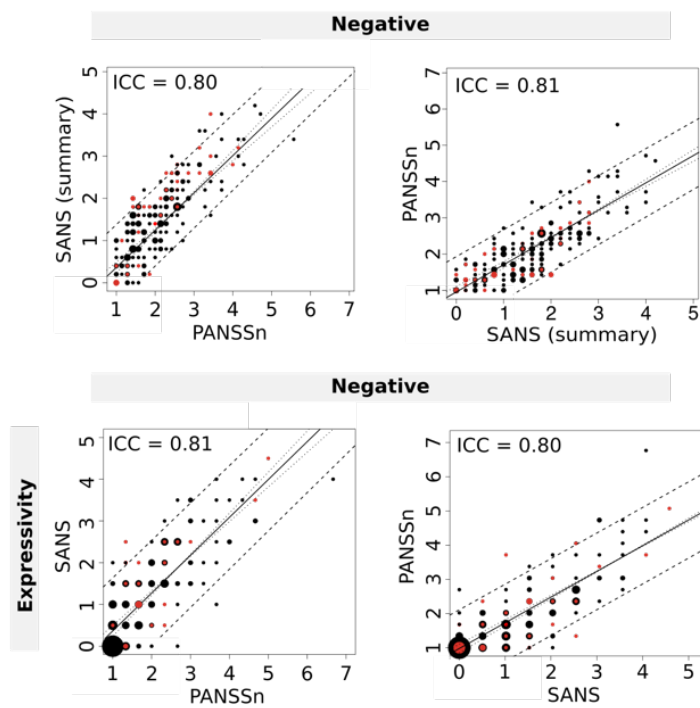

Negative
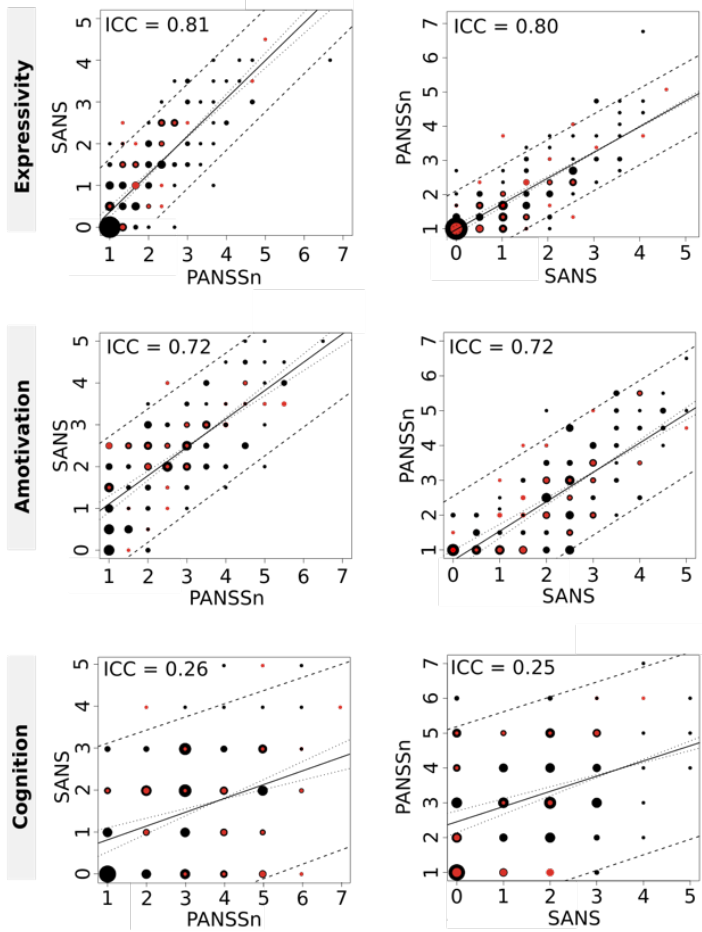

Fig. 2. Linear regression plots. Representative models with an ICC between training and test samples closest to the median ICC for 100 random sampling schemes are shown. Dots are shown in black for training data and red for test data, with larger circles reflecting more data as a function of the square root of the number of observations. 


\section{References}

1. Lyne JP, Kinsella A, O'Donoghue B. Can we combine symptom scales for collaborative research projects? J Psychiatr Res. 2012;46:233-8.

2. Wang L, Alpert KI, Calhoun VD, Cobia DJ, Keator DB, King MD, et al. SchizConnect: Mediating neuroimaging databases on schizophrenia and related disorders for large-scale integration. Neuroimage. 2016;124:1155-67.

3. Kay SR, Fiszbein A, Opler LA. The positive and negative syndrome scale (PANSS) for schizophrenia. Schizophr Bull. 1987;13:261-76.

4. Andreasen N. Scale for the assessment of positive symptoms (SAPS). Iowa city; 1984.

5. Andreasen N. Scale for the assessment of negative symptoms (SANS). Iowa city; 1983.

6. Norman RM, Malla AK, Cortese L, Diaz F. A study of the interrelationship between and comparative interrater reliability of the SAPS, SANS and PANSS. Schizophr. Res. 1996;19:73-85.

7. van Erp TGM, Preda A, Nguyen D, Faziola L, Turner J, Bustillo J, et al. Converting positive and negative symptom scores between PANSS and SAPS/SANS. Schizophr. Res. 2014;152:289-94.

8. Cicchetti DV. Guidelines, criteria, and rules of thumb for evaluating normed and standardized assessment instruments in psychology. Psychological Assesment. 1994;6:284-90.

9. Crow TJ. Molecular pathology of schizophrenia: more than one disease process. Br Med J. 1980;280:66-8.

10. Liddle PF. The symptoms of chronic schizophrenia. A re-examination of the positivenegative dichotomy. Br J Psychiatry. 1987;151:145-51.

11. Ventura J, Thames AD, Wood RC, Guzik LH, Hellemann GS. Disorganization and reality distortion in schizophrenia: A meta-analysis of the relationship between positive symptoms and neurocognitive deficits. Schizophr. Res. 2010;121:1-14.

12. Kelley ME, van Kammen DP, Allen DN. Empirical validation of primary negative symptoms: independence from effects of medication and psychosis. AJP. 1999;156:40611.

13. Marder SR, Galderisi S. The current conceptualization of negative symptoms in schizophrenia. World Psychiatry. 2017;16:14-24.

14. Keator DB, van Erp TGM, Turner JA, Glover GH, Mueller BA, Liu TT, et al. The Function Biomedical Informatics Research Network Data Repository. Neuroimage. 2016;124:1074-9. 
15. First MB, Spitzer RL, Gibbon ML, Williams JBW. Structured Clinical Interview for DSM-IV-TR Axis I Disorders - Patient Edition (SCID-I/P, 11/2002 revision). New-York; 2002.

16. Preda A, Nguyen DD, Bustillo JR, Belger A, O'Leary DS, McEwen S, et al. A positive take on schizophrenia negative symptom scales: Converting scores between the SANS, NSA and SDS. Schizophr. Res. 2018;201:113-9.

17. Leucht S, Kissling W, Davis JM. The PANSS Should Be Rescaled. Schizophr Bull. 2010;36:461-2.

18. R Core Team. R: A Language and Environment for Statistical Computing. Vienna, Austria; 2019. Available from: https://www.R-project.org/

19. Revelle W. psych: Procedures for Personality and Psychological Research. Evanston, Illinois; 2018. Available from: https://CRAN.R-project.org/package=psych

20. Rathbone A, Shaw S, Kumbhare D. ICC.Sample.Size: Calculation of Sample Size and Power for ICC. 2015. Available from: https://CRAN.Rproject.org/package=ICC.Sample.Size

21. American Psychiatric Association. Diagnostic and statistical manual of mental disorders (5th ed., DSM-5). Arlington, VA: American Psychiatric Association; 2013.

22. Wallwork RS, Fortgang R, Hashimoto R, Weinberger DR, Dickinson D. Searching for a consensus five-factor model of the Positive and Negative Syndrome Scale for schizophrenia. Schizophr. Res. 2012;137:246-50.

23. Kotov R, Foti D, Li K, Bromet EJ, Hajcak G, Ruggero CJ. Validating dimensions of psychosis symptomatology: Neural correlates and 20-year outcomes. J Abnorm Psychol. 2016;125:1103-19.

24. Peralta V, Cuesta MJ. How many and which are the psychopathological dimensions in schizophrenia? Issues influencing their ascertainment. Schizophr. Res. 2001;49:269-85.

25. Wing JK, Babor T, Brugha T, Burke J, Cooper JE, Giel R, et al. SCAN. Schedules for Clinical Assessment in Neuropsychiatry. Arch. Gen. Psychiatry. 1990;47:589-93.

26. Russo M, Levine SZ, Demjaha A, Di Forti M, Bonaccorso S, Fearon P, et al. Association Between Symptom Dimensions and Categorical Diagnoses of Psychosis: A Cross-sectional and Longitudinal Investigation. Schizophr Bull. 2013;40:111-9.

27. Barch DM, Bustillo J, Gaebel W, Gur R, Heckers S, Malaspina D, et al. Logic and justification for dimensional assessment of symptoms and related clinical phenomena in psychosis: relevance to DSM-5. Schizophr. Res. 2013;150:15-20. 
28. Foussias G, Agid O, Fervaha G, Remington G. Negative symptoms of schizophrenia Clinical features, relevance to real world functioning and specificity versus other CNS disorders. European Neuropsychopharmacology. 2014;24:693-709.

29. Reichenberg A. The assessment of neuropsychological functioning in schizophrenia. Dialogues Clin Neurosci. 2010;12:383-92.

30. Green MF, Horan WP, Lee J. Nonsocial and social cognition in schizophrenia: current evidence and future directions. World Psychiatry. 2019;18:146-61.

31. Blanchard JJ, Cohen AS. The Structure of Negative Symptoms Within Schizophrenia: Implications for Assessment. Schizophr Bull. 2006;32:238-45.

32. Hawley CJ, Gale TM, Smith VR, Sen P. Depression rating scales can be related to each other by simple equations. Int J Psychiatry Clin Pract. 1998;2:215-9.

33. Hawley CJ, Gale TM, Smith PSJ, Jain S, Farag A, Kondan R, et al. Equations for converting scores between depression scales (MÅDRS, SRS, PHQ-9 and BDI-II): good statistical, but weak idiographic, validity. Hum Psychopharmacol. 2013;28:544-51.

34. Fernandez-Garcimartin H, Bagney A, Moreno-Ortega M, Dompablo M, Torio I, Lobo A, et al. Is it possible to combine different psychotic symptom scales in bipolar disorder? Psychiatry Res. 2014;220:1090-3.

35. Overall JE, Gorham DR. The Brief Psychiatric Rating Scale. Psychol. Rep. 1962;10:799-812.

36. Heckers S, Barch DM, Bustillo J, Gaebel W, Gur R, Malaspina D, et al. Structure of the psychotic disorders classification in DSM-5. Schizophr. Res. 2013;150:11-4. 